in the sample can be detected by techniques such as northern analysis, PCR and microarrays. But how do you know what you're looking for? Much of the groundwork in miRNA identification has been laid by largescale genomics projects that used computational techniques to predict miRNA genes followed by cloning and validation of the predicted sequence. The public miRNA registry currently holds around 1,650 entries for published predicted miRNAs. The big projects now under way are to determine which genes the miRNAs are targeting (see 'Big tasks for small molecules', page 991).

Northern analysis is still the standard for detecting and quantifying miRNA expression. "Northern blotting, even if time consuming, is by far the best technique to study miRNA expression because of its sensitivity and quantitativity," says Jiahuai Han at the Scripps Research Institute, La Jolla, California, who is looking at the mechanisms by which miRNAs affect the stability of their target mRNAs. "Primer extension has the advantage of being quicker but, unfortunately, is less quantitative," he says.

Integrated DNA Technologies (IDT) of Coralville, Iowa, sells miRNA tools to increase the sensitivity of northern analysis. Its StarFire kit for probe labelling makes labels composed of $10^{32} \mathrm{P}$-alpha-dATPs rather than the more usual single ${ }^{32} \mathrm{P}$-gamma-ATP. "We use a special template and reaction conditions that give a 10-base tail with almost no heterogeneity," explains Mark Behlke, vice president of molecular genetics at IDT, "so all probe molecules are the same - it is very different from other tailing procedures." Manufacturers are also gearing up to make miRNA-specific probes; miRCURY LNA (locked nucleic acid) detection probes for all known miRNAs are available from Exiqon of Vedbaek, Denmark, for example, and can be used for in situ hybridization, northern analysis, PCR and gene knockdown.

Another choice for miRNA detection is Ambion's mirVana miRNA detection kit. Ambion claims that its assay is $100-500$ times more sensitive than northern analysis, as the radiolabelled probes are hybridized in solution instead of on a membrane as in northern blotting. The company claims that this method gives the researcher a better shot at detecting and quantifying low-abundance miRNAs because the probe and target have more opportunities to bind when in solution.

Taking the PCR road, Applied Biosystems of Foster City, California, is soon to launch a new TaqMan microRNA assay for miRNA detection and quantitation, which the company claims will detect only mature miRNAs and not precursors. According to Marcum Bell, product manager of gene-expression assays at Applied Biosystems, the assay "uses specific stem-looped primers for reverse transcription of the mature miRNA, followed by quantitative real-time PCR." A claimed advantage of the new assay is its wide dynamic range of up to 7 log units, enabling detection of both lowand high-abundance miRNAs.

For an alternative to PCR-based miRNA assays, US Genomics of Woburn, Massachu- setts, recently unveiled its Trilogy 2020 Single Molecule Analyzer for the high-throughput detection and quantitation of single molecules of nucleic acid without amplification. The Trilogy 2020 can be used along with the company's Direct miRNA Assays for miRNA work. The assay includes two fluorescently tagged probes (tags can be red, blue or green) that are designed to hybridize to the miRNA of interest. Specificity relies on the very high likelihood that only the target miRNA will 兽 hybridize to both probes. After hybridization, $s$

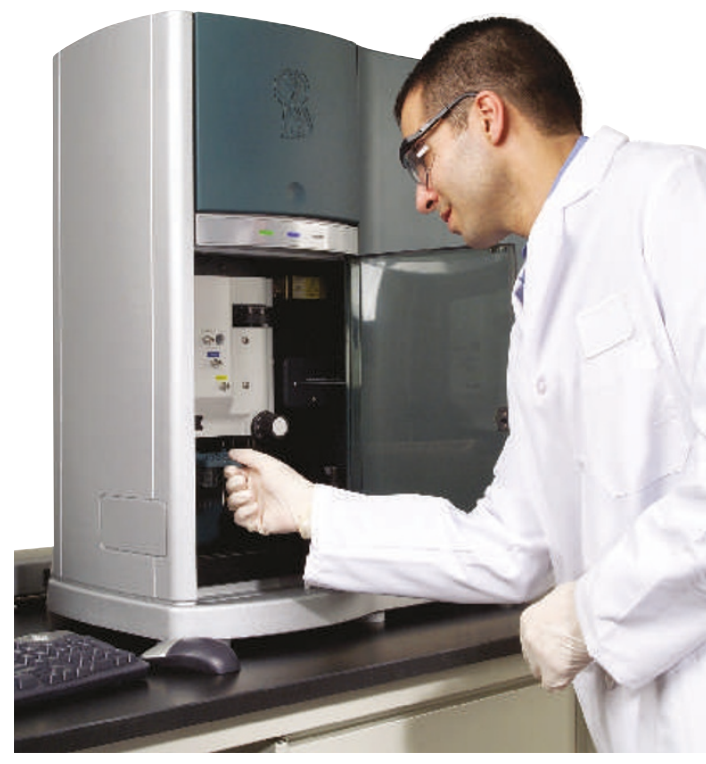

The Trilogy Single Molecule Analyzer can be used for miRNA detection.

\title{
GENOTYPING GETS UP TO SPEED
}

At the high-throughput end of multiplex SNP genotyping, Illumina of San Diego, California, is currently beta testing the Sentrix Human-1 BeadChip, containing more than 100,000 SNPs, nearly 30,000 of which are located in genes, with another 40,000 within $10 \mathrm{~kb}$ of genes. The company is developing BeadChips containing 250,000 and 500,000 SNPs for release next year, which will make it possible to genotype 1 million SNPs on just a pair of chips.

Using a different approach to SNP genotyping, the LightTyper Genotyping System from Roche Applied Science of Indianapolis, Indiana, is designed for the heavyduty end of the market, where thousands of samples may have to be genotyped each day. After PCR amplification of genomic samples in 96- or 384-well plates using a standard thermal cycler, plates are transferred directly to the Light Typer and genotyped within
10 minutes, using the melting points of fluorescently labelled probes hybridized with the SNPs as the detection system. Probetarget complexes with different melting points reflect the presence of different alleles, and show up as allele-specific peaks in the melting curves. Because many samples can be tested simultaneously, "the LightTyper instrument is mainly

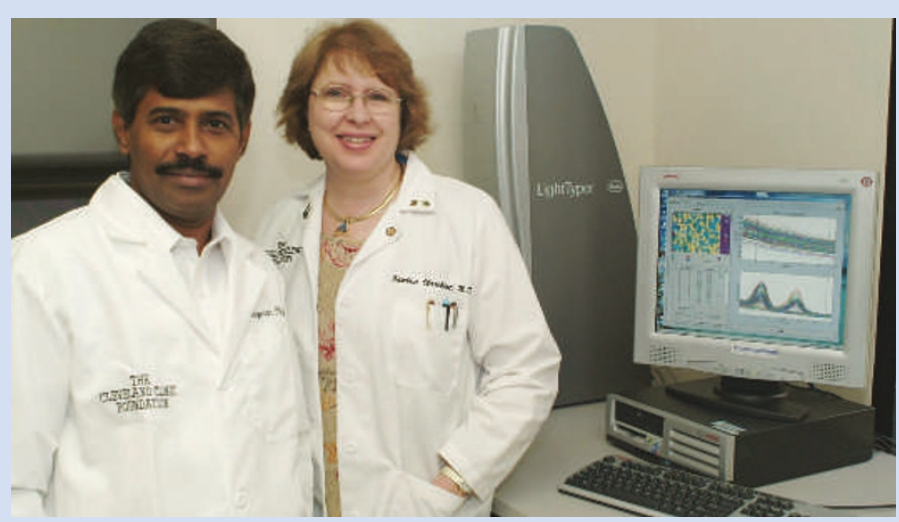

Roche's LightTyper speeds up high-throughput genotyping.

used for SNP genotyping, in particular for disease association studies," says Burkhard Ziebolz of science communications at Roche Diagnostics in Mannheim, Germany.

The Luminex xMAP platform for multiplex genotyping is used by several genetic diagnostics service companies, including TmBioscience of Toronto, Ontario, which has developed the first Food and Drug Administrationapproved multiplexed test for cystic fibrosis mutations, and Tepnel LifeCodes of Manchester, UK, whose speciality is HLA DNA typing.

For less-intensive SNP detection, the READIT SNP genotyping system from Promega of Madison, Wisconsin, can be scaled up or down. It uses the company's READase-mediated destabilization of perfectly matched probe-target complexes coupled with a luciferase reporter assay for the ATP generated. With appropriately designed probes, the system can detect SNPs, insertions, deletions and chromosomal translocation, and can estimate allele frequency and carry out allele-correlation studies. And PerkinElmer of Boston, Massachusetts, have SNP detection kits in their established AcycloPrime range. 